\title{
Methodology for immunotropic activity study of humic acids
}

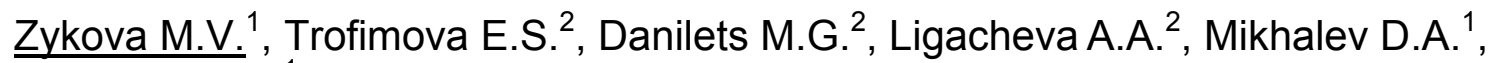 \\ Belousov M.V. ${ }^{1}$ \\ ${ }^{1}$ Siberian State Medical University, Tomsk, Russia, huminolog@mail.ru \\ ${ }^{2}$ Goldberg Research Institute of Pharmacology and Regenerative Medicine, Tomsk, Russia
}

doi: 10.36291/HIT.2019.zykova.019

Investigation of the humic acids (HAs) effect on various factors of cellular and humoral immunity is a currently important scientific field in immunopharmacology. We studied of immunomodulating properties for peat HAs. For HAs isolation, nine representative types of peat were taken from major peat bogs in the Tomsk region (Russia). The nine peat samples analyzed varied in the botanical composition, the degree of decomposition (from 5-10\% to 40$45 \%$ ), and the ash content (from $2.6 \%$ to $16.3 \%$ ). The $\mathrm{HA}$ fractions isolated as described earlier [Zykova, 2018] using basic $(\mathrm{NaOH})$ and pyrophosphate $\left(\mathrm{Na}_{4} \mathrm{P}_{2} \mathrm{O}_{7}\right)$ extractions, were designated as HAb and HAp, respectively. Methodology for immunotropic activity study of HAs consisted of several stages.

First step was the screening of HAs NO-stimulating properties (18 samples) of various concentrations $(0.1 ; 1 ; 10 ; 50$ and $100 \mu \mathrm{g} / \mathrm{mL})$ in murine peritoneal macrophages cultures. All tested HAs exhibited stimulation of NO production. It was stated that HAp samples have higher activity than HAb samples. The second step consisted of two experiments that were carried out in murine peritoneal macrophages culture in comparison with lipopolysaccharide (LPS) as stimulated control and the standard LPS inhibitor antibiotic Polymyxinum B that blocks stimulating activity of LPS by binding with the endotoxin. Results showed that HAp samples are more preferable in activation of macrophages than HAb samples due to their immunotropic properties being independent from endotoxin presence. Absence of endotoxin in natural substance is preferable in development of immunotropic medications. The HAp samples of raised peats induced cells activation by classical path, increased NO production and reduced arginase expression. Results allowed to determining the most active HAp sample from the raised pine-cotton-grass peat (aHAp) as with addition of the LPS inhibitor in the cell culture its NO-stimulating activity did not decrease, but increased, arginase activity decreased. This indicates that aHAp sample have potential immunotropic activity mediated by Th1-dependent immune response. The third step was a study of the cytokine profile for aHAp sample that was carried out using key cytokines: IL-2, IL-4, IL-10, IL-12, TNF- $\alpha$ and INF-y. The results showed that the aHAp sample reduced the production of IL-10 against the background of stimulation of the production of key proinflammatory cytokines IL-2, IL-12, TNF- $\alpha$ and INF- $y$ by antigen-presenting cells. Thus, the aHAp sample showed the properties of a classic M1 activator, capable of supporting and enhancing the Th1 immune response. The fourth step was to study the effect of an aHAp sample on the reactions of the Th1 and Th2-dependent immune response in vivo. To assess the Th1-immune response, a mice immunization model was used by ram erythrocytes. The effect of the aHAp sample on the humoral immunity was evaluated by the number of antibody-forming cells in the spleen and the synthesis of hemagglutinins. The effect of the aHAp sample on the cellular immunity was evaluated by the delayed-type hypersensitivity reaction (this is a Th1-marker reaction). The course of administration of the aHAp sample reduced the intensity of the cellular immunity reaction and enhanced the indices of humoral immunity. This means that the aHAp sample really showed the properties of a classic M1 activator, meaning it can stimulate the immune response in infectious and inflammatory processes, chronic and oncological diseases. To evaluate the Th2-dependent immune response, an ovalbumin model of animal immunization was used. We investigated the level of $\lg \mathrm{G} 1$ and $\operatorname{lgE}$, degranulation reaction of mast cells and anaphylactic index by Weigle. The aHAp sample was found to the active inhibition of the synthesis of IgG1 and IgE, stabilization of mast cell membranes and suppress Th2-dependent immune response. This means that the aHAp sample has an antiallergic effect. Thus, HAs are promising immunomodulators. 